\title{
PREDICTION OF LAMINAR-TURBULENT TRANSITION ON AN AIRFOIL AT HIGH LEVEL OF FREE-STREAM TURBULENCE
}

\author{
V. Chernoray \\ Department of Applied Mechanics, Chalmers University of Technology \\ Göteborg 412 96, Sweden
}

\begin{abstract}
Prediction of laminar-turbulent transition at high level of free-stream turbulence in boundary layers of airfoil geometries with external pressure gradient changeover is in focus. The aim is a validation of a transition model for transition prediction in turbomachinery applications. Numerical simulations have been performed by using a transition model by Langtry and Menter for a number of different cases of pressure gradient, at Reynolds-number range, based on the airfoil chord, 50000 $\leq \mathrm{Re} \leq 500000$, and free-stream turbulence intensities $2 \%$ and $4 \%$. The validation of the computational results against the experimental data showed good performance of used turbulence model for all test cases.
\end{abstract}

\section{INTRODUCTION}

Components of an aeroengine operate in a Reynolds-number range between $5 \cdot 10^{4}$ and $1 \cdot 10^{6}[1]$. For this range of Reynolds numbers, the laminar-turbulent transition in a boundary layer and separation play an important role in determining the flow and heat transfer. In order to predict the aerodynamic and heat transfer performance of aeroengine components accurately, one needs to predict the location and length of the regions with laminar-turbulent transition and separation. This prediction is a challenging task due to the presence of varying pressure gradients and high level of free-stream turbulence.

Except for the fan, the level of turbulence in an aeroengine is high, above $1 \%$, and classic transition scenario is seldom realized. The typical transition regimes are the bypass transition and separated flow transition. An overview of different scenarios of laminar-turbulent transition at high free-stream turbulence and presence of pressure gradient can be found in studies [2-6]. On an airfoil, if the Reynolds number is high enough so that the transition is complete before a laminar separation can occur or if the boundary layer does not separate because the flow deceleration is slight, then an attached flow transition occurs. If the

This is an Open Access article distributed under the terms of the Creative Commons Attribution License 4.0, which permits unrestricted use, distribution, and reproduction in any medium, provided the original work is properly cited. 
adverse pressure gradient is more severe and the Reynolds number is low, then the laminar boundary layer tends to separate and transition occurs in the free shear layer of the separation bubble. This scenario is called a separated flow transition. This type of transition typically may occur near an airfoil leading edge on suction or pressure side, or both, and near the point of minimum pressure on the suction side. At intermediate Reynolds numbers, the transition occurs in the attached flow ahead of the location where the laminar boundary layer would separate. The transition physics can be rather complex and this topic is covered in detail in, e.g., recent monograph by Boiko et al. [7].

According to famous review by Mayle [1], the most important parameters for the onset of transition on an airfoil are the pressure gradient and incoming flow turbulence intensity. The effect of the turbulence length scale is also substantial but can be incorporated into the turbulence intensity by introducing a well-known scaling [1]. Surface roughness, heat transfer, compressibility, and wall curvature are the secondary effects, which typically have an order of magnitude weaker influence on transition as compared to the primary effects. The most debated is the influence of such parameter as surface curvature. Mayle [1] concludes that the curvature effect in turbomachinery components applies only to convex surfaces and that even for convex surfaces, the onset of transition at high free stream turbulence virtually identical to that for a flat plate.

If the component has to operate over a wide range of Reynolds numbers and angles of incidence, all above scenarios can take place at the different flow regimes. Such situations are particularly difficult to model numerically.

A number of turbulence models claim a possibility of transition prediction but none of them is proven to be flawless so far. A transition model suggested by Menter et al. [8] is based on correlation-based approach which appears to provide consistent results. This model has been applied to a number of two(2D) and three-dimensional (3D) test flow cases [9] and simulations agreed well with experiments for the studied cases at a wide range of Reynolds numbers and freestream turbulent intensities. However, a literature survey shows that not so many other validation cases of this model are publicly available. To fulfil this gap and to validate the applicability of model by Langtry and Menter for the cases with different pressure gradient, turbulence intensity and Reynolds number is the main purpose of current study.

\section{EXPERIMENTAL AND NUMERICAL SETUP}

\subsection{Airfoil Models}

Current experiments were particularly designed for validation of boundary layer transition and for detailed measurement of the boundary layer development on an airfoil. In order to provide the measurements of boundary layers with good 
spatial resolution, large-scale airfoil test models were used. Several different airfoil geometries were selected to model the effect of different pressure gradient distributions. Since the range of flow Reynolds numbers of interest for current study is $5 \cdot 10^{4}-5 \cdot 10^{5}$ (based on the chord) and the incoming flow turbulence intensity is above $2 \%$, the aft part of the airfoil was obviously not of major interest. Thus, truncated airfoil models with the aft part replaced by a straight section (Fig. 1) were designed and manufactured. This design is advantageous due to a simpler manufacturing and makes the near-wall measurements easier and more reliable.

Figure 1 shows the pressure distributions for a modified model with straight aft part and for a full airfoil and the corresponding geometries are superimposed. One can observe that for the modified model, the magnitude of the pressure peak is decreased by $25 \%$ and the magnitude of the favourable pressure gradient in the accelerated region is decreased correspondingly, while the adverse pressure gradient becomes stronger directly

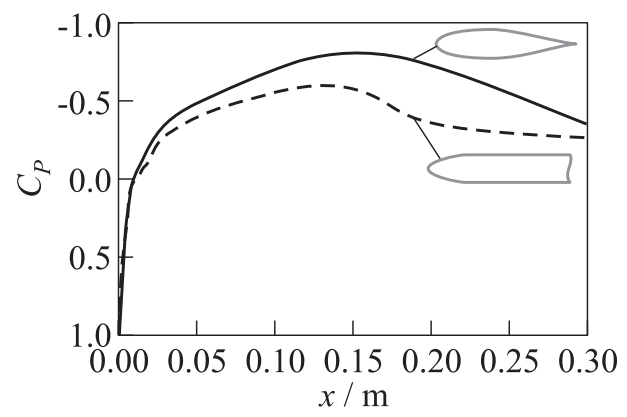

Figure 1 Comparison of pressure distributions on a full airfoil and on airfoil model with straight aft part. For the latter, the straight section starts at $x=0.16 \mathrm{~m}$ after the pressure minimum with a zone of a milder gradient further downstream. Apart from these differences, the pressure distribution for the modified model is a typical airfoil-like pressure distribution with characteristic pressure gradient changeover.

To cover a range of pressure gradient distributions typical for turbomachinery applications, four different airfoil nose shapes were developed. The geometry of the models and corresponding pressure distributions obtained numerically are shown in Fig. 2. The model shapes were designed based on NACA6 airfoil of two different modifications and with two different aspect ratios. Cases 1 and 2 are, respectively, the factor of two thickened versions of Cases 3 and 4. Correspondingly, the pressure minimum for these models is twice larger in absolute value compared to Cases 3 and 4 (the values are -1.2 and -0.6 , respectively). Cases 1 and 3 both have the location of pressure minimum shifted towards the leading edge which results in a stronger favourable pressure gradient and slightly milder adverse pressure gradient compared to Cases 2 and 4 . Overall, the favourable pressure gradient is the strongest for Case 1 and gradually decreasing for Cases 2, 3 , and 4 .

Current Cases 1 and 2 model the pressure distribution of high- and mediumloaded turbomachinery vanes (cf. [10]) and Cases 3 and 4 have typical pressure distributions for low-loaded vanes [11]. 


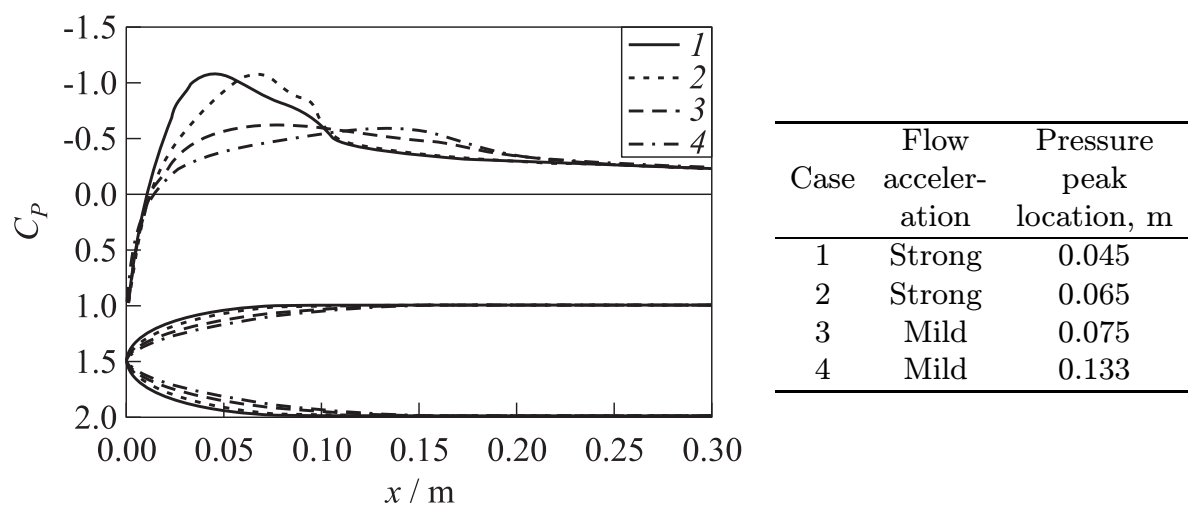

Figure 2 Pressure distributions for four studied airfoil models: 1 - Case $1 ; 2-$ Case 2; 3 - Case 3 ; and 4 - Case 4

\subsection{Experimental Setup}

The experiments were performed in a wind tunnel facility at Chalmers and experimental setup is depicted in Fig. 3. The used tunnel was of open circuit type and was operated at velocities between 5 and $18 \mathrm{~m} / \mathrm{s}$. The cross section of the facility is $200 \times 1200 \mathrm{~mm}$. Since the thickness of the airfoil models was $100 \mathrm{~mm}$, the blockage of the wind-tunnel was $8 \%$. Note, however, that all presented CFD results were obtained with the blockage effect taken into account since the calculations were performed for the same size of the numerical wind tunnel as in experiments; thus, the blockage had no influence on the discussed results.

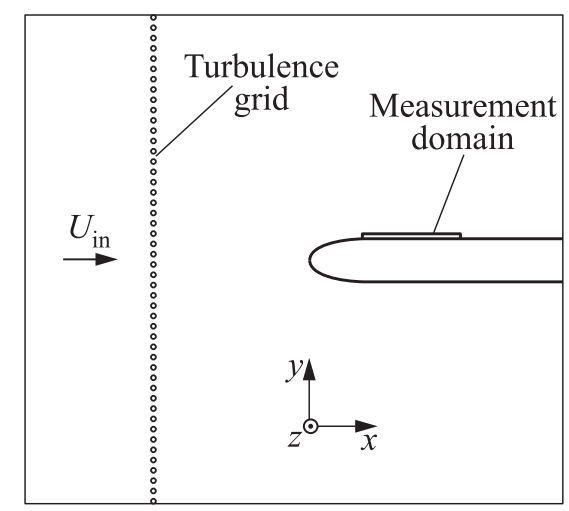

Figure 3 Experimental setup 
In the wind tunnel, the test section was equipped with an end-wall boundarylayer suction system for removal of sidewall boundary layers, which allowed obtaining the flow with good two-dimensionality.

The airfoil test models are manufactured by using a stereolithography (SLA) technique which has a typical accuracy of $\pm 0.1 \%$ of the model size. The models produced by this method have small traces on the surface due to a finite resolution of the SLA machine. For obtaining smooth surfaces, an epoxy coating was applied on the models and thereafter, the surfaces were polished.

For monitoring of the static pressure distributions, each model was equipped with 15 pressure taps distributed along the streamwise direction.

A turbulence grid is used to create an elevated turbulence intensity of the inflow. The grid is built of 5-millimeter bars of circular cross section and a mesh size of the grid, $M$, is $25 \mathrm{~mm}$, thus giving a porosity (ratio between open and total area of the grid) of 0.64. A 1-millimeter thick wire was additionally spirally coiled around the grid bars in order to decrease the mean-velocity modulation in the wakes behind the bars. The grid was placed at $800 \mathrm{~mm}$ upstream of the airfoil model $(x / M=32)$ in configuration of $2 \%$ incoming flow turbulence intensity and at $270 \mathrm{~mm}$ upstream of the model $(x / M=11)$ in the case of 4 percent turbulence intensity. The turbulence intensity was measured at the model leading edge. The turbulence length scale and turbulence anisotropy generated by this grid were evaluated earlier [10] by using cross-hot-wire measurements. It is of importance to note that in current computational fluid dynamics (CFD) simulations, the turbulence intensity and turbulence length scale were adjusted at the inlet of CFD domain to match the both respective values in CFD and experiment at the airfoil leading edge.

The boundary layer measurements were performed by using a constant temperature hot-wire anemometer. A single-wire probe was used with a tungsten wire of 3-millimeter length and $5 \mu \mathrm{m}$ in diameter. The probe calibration was performed in a dedicated calibrator. The maximum error in the probe calibration was within $0.5 \%$ for all calibration points. Probe positioning and data acquisition were fully automated. A three-axis probe positioning system had a resolution of $1.6 \mu \mathrm{m}$. The accuracy of finding the distance between the hot-wire and wall was $50 \mu \mathrm{m}$. The boundary layer profiles were measured at the mid-span at 16 streamwise locations with 27 points in each profile. The measurements were repeated for each of 18 investigated flow cases.

The inlet flow velocity in the wind tunnel was monitored by a Pitot-Prandtl tube connected to a digital micromanometer which had sensors for temperature and absolute pressure readings. Surface static pressure distributions were measured by a 16-channel PSI 9116 digital pressure scanner (Pressure System Inc.) which had a measuring range of $\pm 2500 \mathrm{~Pa}$. The accuracy of the scanner in the measurement range of current experiment $( \pm 250 \mathrm{~Pa})$ is $\pm 2 \mathrm{~Pa}$. The measured surface pressure distributions agreed well with the numerical distributions shown in Fig. 2. The curve shape and the location of pressure minimum were captured 
well by $\mathrm{CFD}$ and the magnitude of the pressure minimum has agreed within $10 \%$ between CFD and experiments. These results can be found in an earlier work [12].

\subsection{Numerical Setup}

Numerical calculations were performed with a Gamma-Theta transition turbulence model by Menter et al. [8]. This correlation-based model uses transport equations for intermittency and momentum thickness Reynolds number. The intermittency equation is coupled with Menter's $k-\omega$ shear stress transport (SST) model and used to turn on the production of the turbulent kinetic energy beyond the turbulent transition region. The second transport equation is formulated in terms of the momentum thickness Reynolds number at the transition onset. An empirical correlation is used to control the transition onset criteria in the intermittency equation.

In the current study, the steady 2D Reynolds-averaged Navier-Stokes (RANS) computations were performed by using Ansys Fluent 12 pressure-based implicit finite volume solver and second-order discretization scheme. The computational grid consists of an O-grid surrounding the model, which is shown in Fig. 4 by a black color. The grid in the free stream consists of a block with quadrilateral cells (a light-grey region in Fig. 4) and a block with unstructured grid which is shown by a dark-grey colour.

The baseline grid has $10^{5}$ cells with the first row of cells at $y^{+}<0.5$. For the grid independency check, two additional grids, a refined and coarsened, were used. The refined grid uses $7 \cdot 10^{5}$ cells (with $y^{+}<0.25$ ) and the coarsened grid has the same number of cells as the baseline grid but with the O-grid part coarsened in such a way that the grid $y^{+}$values are below 1 . The calculations on three different grids were performed for 6 of the presented numerical cases.

The boundary conditions in numerical calculations were carefully matched with the corresponding conditions from the wind-tunnel tests. The turbulence intensity and turbulent length scale at the inlet were adjusted so that at the airfoil leading edge, the values were equal in CFD and in experiments.

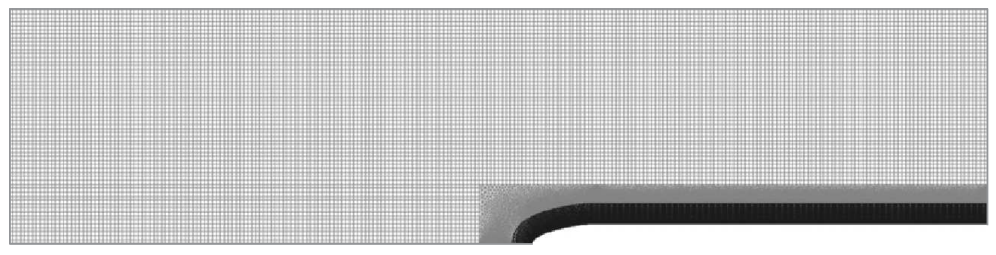

Figure 4 Computational grid 


\subsection{Numerical Method}

The $k-\omega$ SST model differs from the standard $k-\omega$ model in a few major modifications: gradual change from the standard $k-\omega$ model in the inner region of the boundary layer to a high-Reynolds-number version of the $k-\varepsilon$ model in the outer part of the boundary layer, presence of a damped cross-diffusion derivative term in the $\omega$ equation, modified turbulent viscosity formulation to account for the transport effects of the turbulent shear stress, and different model constants. The $k-\omega$ SST model benefits from the robust and accurate formulation of the $k-\omega$ model in the near-wall region with the free-stream independence of the $k-\varepsilon$ model in the far field. These features make it more reliable in adverse pressure gradient and separating flows than the standard $k-\omega$ model. The equations for $k$ and $\omega$ are written as:

$$
\begin{aligned}
& \frac{\partial}{\partial t}(\rho k)+\frac{\partial}{\partial x_{i}}\left(\rho k u_{i}\right)=\frac{\partial}{\partial x_{j}}\left(\Gamma_{k} \frac{\partial k}{\partial x_{j}}\right)+\widetilde{G_{k}}-Y_{k}+S_{k} \\
& \frac{\partial}{\partial t}(\rho \omega)+\frac{\partial}{\partial x_{i}}\left(\rho \omega u_{i}\right)=\frac{\partial}{\partial x_{j}}\left(\Gamma_{\omega} \frac{\partial \omega}{\partial x_{j}}\right)+G_{\omega}-Y_{\omega}+D_{\omega}+S_{\omega}
\end{aligned}
$$

where $\Gamma_{k}$ and $\Gamma_{\omega}$ represent the effective diffusivity of $k$ and $\omega ; \widetilde{G_{k}}$ represents the generation of turbulence kinetic energy due to mean velocity gradients and $G_{\omega}$ represents the generation of $\omega ; Y_{k}$ and $Y_{\omega}$ represent the dissipation of $k$ and $\omega$ due to turbulence; $S_{k}$ and $S_{\omega}$ are the user-defined source terms; and $D_{\omega}$ represents the cross-diffusion term which role is to blend the standard $k-\omega$ and $k-\varepsilon$ models.

The transition $k-\omega$ SST model is based on the coupling of the SST transport equations with two additional transport equations, one for the intermittency, $\gamma$, and one for the transition onset criteria, which is the momentumthickness Reynolds number, $\widetilde{\operatorname{Re}_{\theta} t}$. These additional equations do not attempt to model the physics of transition, and are correlation-based. The equations are written as

$$
\begin{gathered}
\frac{\partial(\rho \gamma)}{\partial t}+\frac{\partial\left(\rho U_{j} \gamma\right)}{\partial x_{j}}=P_{\gamma 1}-E_{\gamma 1}+P_{\gamma 2}-E_{\gamma 2}+\frac{\partial}{\partial x_{j}}\left[\left(\mu+\frac{\mu_{t}}{\sigma_{\gamma}}\right) \frac{\partial \gamma}{\partial x_{j}}\right] ; \\
\frac{\partial\left(\rho \widetilde{\operatorname{Re}_{\theta t}}\right)}{\partial t}+\frac{\partial\left(\rho U_{j} \widetilde{\operatorname{Re}_{\theta t}}\right)}{\partial x_{j}}=P_{\theta t}+\frac{\partial}{\partial x_{j}}\left[\sigma_{\theta t}\left(\mu+\mu_{t}\right) \frac{\partial \widetilde{\operatorname{Re}_{\theta t}}}{\partial x_{j}}\right]
\end{gathered}
$$

In the above equations, $\sigma_{\gamma}$ and $\sigma_{\theta t}$ are the constants; $\mu$ and $\mu_{t}$ represent the molecular and turbulent viscosity, respectively; and $P_{\gamma 1}$ and $E_{\gamma 1}$ are the transition sources, which depend upon the local strain rate and empirical correlations for the transition length and transition onset. These correlations provide the connection between the critical Reynolds number $\mathrm{Re}_{c}$, where the intermittency first starts to increase in the boundary layer, and the transition Reynolds num- 
ber $\widetilde{\operatorname{Re}_{\theta t}} \cdot P_{\gamma 2}$ and $E_{\gamma 2}$ are the destruction/relaminarization sources, which are the functions of the vorticity magnitude $\Omega$ and ensure that the intermittency remains zero in the laminar boundary layer. The source term, $P_{\theta t}$, in Eq. (3) forces the transported scalar $\widetilde{\operatorname{Re}_{\theta t}}$ to match the local value of $\operatorname{Re}_{\theta t}$ calculated from an empirical correlation outside the boundary layer. The interaction of the transition model with the SST turbulence model is handled by modifying the production/generation, $\widetilde{G_{k}}$, and destruction/dissipation, $Y_{k}$, terms in the $k$ equation (1), but keeping the corresponding terms unchanged in the $\omega$ equation (2). The successful interaction entails one more change which is in the blending function, used for switching between the $k-\omega$ and $k-\varepsilon$ models [8].

\section{RESULTS}

In this paper, the results are presented from 9 test cases of total 18, the rest of experimental and numerical data can be found in report [12].

In Fig. 5, a set of profiles of mean velocity and turbulence intensity are shown for Case 4 at inlet velocity $9 \mathrm{~m} / \mathrm{s}$ and free-stream turbulence intensity $2 \%$. Case 4 is the case of mild pressure gradient and the velocity $9 \mathrm{~m} / \mathrm{s}$ represents the medium of three studied velocities: 5,9 , and $18 \mathrm{~m} / \mathrm{s}$. Note that the profiles are for every third of all measured $x$-stations.

From the mean velocity profiles, it is clearly seen that in this case, the boundary layer is initially laminar and the initial profile at $x=0.13 \mathrm{~m}$ has the shape close to the Blasius velocity profile, which is depicted for comparison. For the next measurement point at $x=0.22 \mathrm{~m}$, the action of adverse pressure gradient

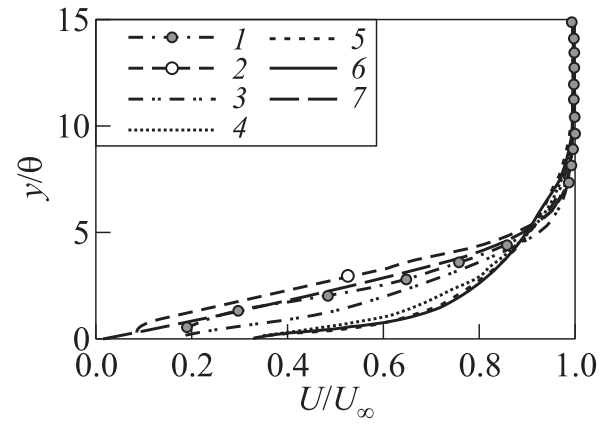

(a)

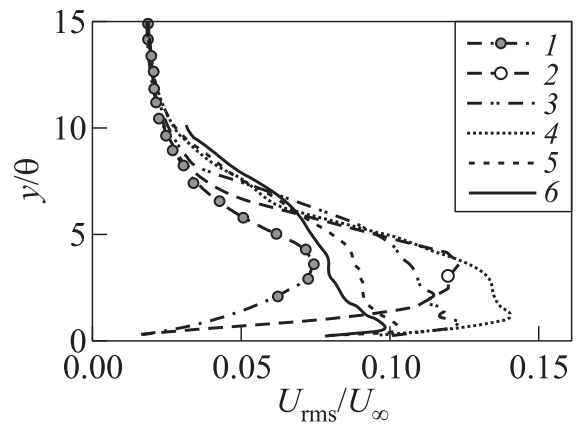

(b)

Figure 5 Boundary layer profiles of mean velocity $(a)$ and turbulence intensity $(b)$ for Case 4 at inlet velocity $U_{\text {in }}=9 \mathrm{~m} / \mathrm{s}$ and inlet turbulence intensity $\mathrm{Tu}_{\mathrm{in}}=2 \%: 1$ $x=0.13 \mathrm{~m} ; 2-0.22 ; 3-0.25 ; 4-0.28 ; 5-0.31 ; 6-x=0.34 \mathrm{~m} ;$ and $7-$ Blasius velocity profile 
results in a less full profile than the Blasius profile. The profile of the turbulence intensity at the first measurement station has a typical distribution for a laminar boundary layer subjected to high turbulence intensity. One can observe that the magnitude of turbulence increases from the free-stream value of $2 \%$ to $8 \%$ inside the boundary layer. This peak value is located roughly in the middle of the boundary layer at $y=3.5 \theta$. At the next streamwise position, $x=0.22 \mathrm{~m}$, the laminar-turbulent transition starts and proceeds until $x=0.28 \mathrm{~m}$. The maximum turbulence intensity near the wall is $14 \%$ in the middle of the transitional range at $x=0.25 \mathrm{~m}$. As seen, from $x=0.25 \mathrm{~m}$, the position of the maximum root mean square (rms) in the boundary layer is shifted towards the wall, and at $x=0.28 \mathrm{~m}$, the shape of the rms profile becomes typical for a turbulent boundary layer. The last two measurement stations, $x=0.31$ and $0.34 \mathrm{~m}$, reveal typical distributions of the mean velocity and turbulence intensity for a turbulent boundary layer with an observable similarity for both the mean and rms velocity profiles.

\subsection{Effect of Reynolds Number}

Figure 6 shows contour plots of turbulence intensity within the boundary layer for Case 4 at different inflow Reynolds numbers. It is seen that the turbulent region in the boundary layer moves upstream as flow Reynolds number increases. The intermittency contours help to highlight the transition start and end. Transition location is where the intermittency is 0.5 . The lowest inlet flow Reynolds number produces the most extensive transitional region as expected.

\subsection{Effect of the Pressure Gradient}

Contour plots in Fig. 7 show the turbulence intensity distributions for two cases of different pressure gradients. Figure $7 a$ shows the measurements for the case of a stronger pressure gradient in Case 2 and Fig. $7 b$ is for Case 4 . One can observe that the transition point moves upstream when a stronger adverse pressure gradient is present. At the same speed and free-stream turbulence level, the effect of increasing the adverse pressure gradient is, as might be expected, an earlier transition beginning.

\subsection{Effect of the Inlet Turbulence Intensity}

Contour plot in Fig. 8 shows the effect of varying free-stream turbulence. The case of elevated turbulence intensity, $\mathrm{Tu}=4 \%$, is shown in comparison with previously discussed case of $\mathrm{Tu}=2 \%$. As expected, higher level of freestream turbulence promotes the transition onset. It is noticeable that in case of $\mathrm{Tu}$ $=4 \%$, the transition begins already in the zone of favourable pressure gradient. 

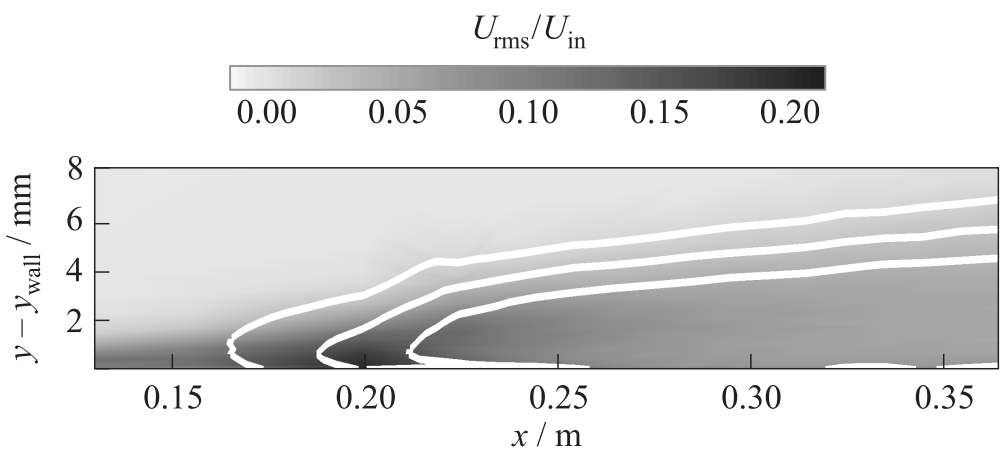

(a)

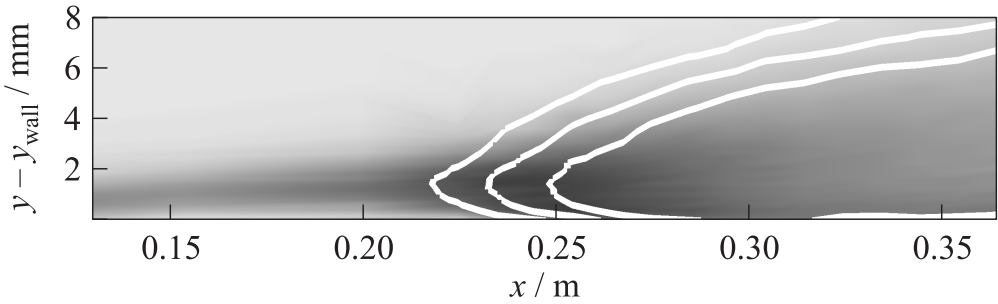

(b)

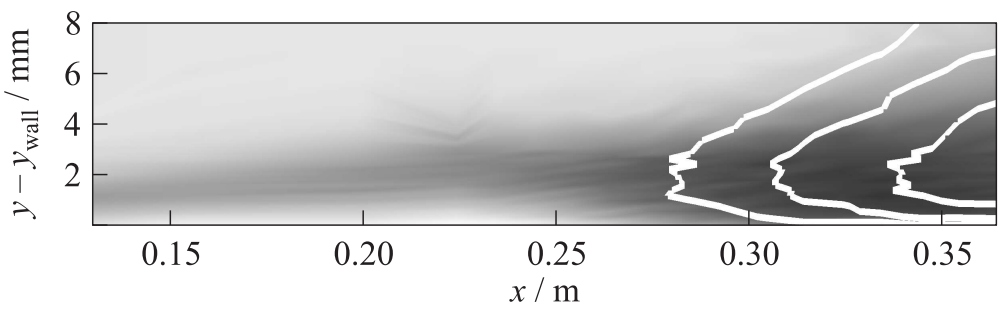

(c)

Figure 6 Effect of inlet Reynolds number on boundary layer transition for the case of mild pressure gradient (Case 4 ) at $\mathrm{Tu}_{\mathrm{in}}=2 \%$. Inlet velocity $U_{\mathrm{in}}=18(a), 9(b)$, and $5 \mathrm{~m} / \mathrm{s}(c)$. Pseudocolors depict the local turbulence intensity $U_{\mathrm{rms}} / U_{\mathrm{in}}$ and white contour lines depict intermittency levels of $0.25,0.5$, and 0.75

\subsection{Validation of computational fluid dynamics results}

In this subsection, the results of CFD calculations are presented. The calculations are validated by the experimental data. For validation, distributions of the momentum thickness Reynolds number are compared with distributions of the shape factor, $H_{12}$.

Figure 9 shows the results of comparison between CFD and experiment for the case of mild pressure gradient (Case 4 ) at turbulence intensity $\mathrm{Tu}_{\mathrm{in}}=2 \%$. 


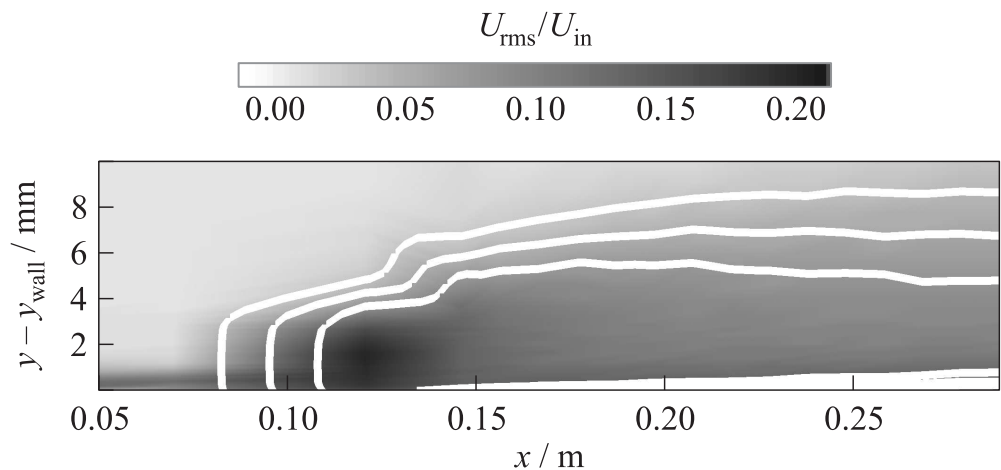

(a)

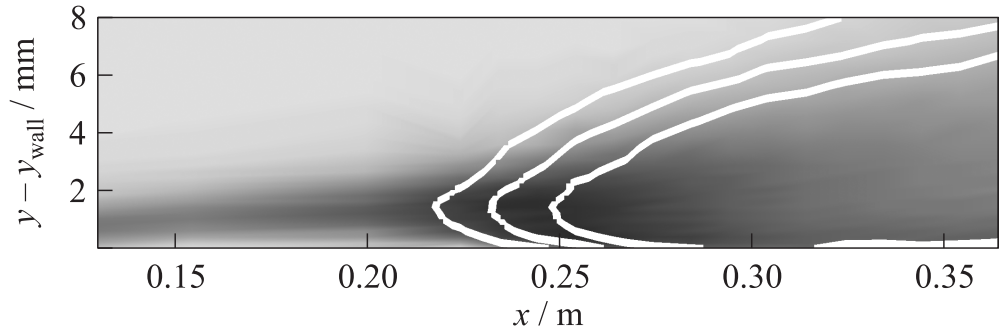

(b)

Figure 7 Effect of the pressure gradient: $(a)$ the case of strong pressure gradient (Case 2) and (b) the case of mild pressure gradient (Case 4) at $U_{\text {in }}=9 \mathrm{~m} / \mathrm{s}$ and $\mathrm{Tu}_{\text {in }}=2 \%$. Note different streamwise axes. Pseudocolors depict local turbulence intensity $U_{\mathrm{rms}} / U_{\mathrm{in}}$ and white contour lines depict intermittency levels of $0.25,0.5$, and 0.75

The results are shown for inlet velocities $U_{\text {in }}=5,9$, and $18 \mathrm{~m} / \mathrm{s}$. The CFD results are shown from calculations on 3 different grids to demonstrate the grid independency. The development of the momentum thickness Reynolds number is captured very well by CFD in all cases of inlet velocity. Observed discrepancies are within $10 \%$ and caused, mainly, by discrepancies in the momentum thickness, since the outer velocity distributions are captured very well by calculations. Note that for two initial measurement locations, $x=0.13$ and $0.15 \mathrm{~m}$, the difference in measured and predicted momentum thickness is $30 \mu \mathrm{m}$ which is attributed to the accuracy of hot-wire positioning with respect to the wall. For the downstream stations, the discrepancy is caused by the underprediction of the transition location in CFD which, in turn, results in differences in the momentum thickness. The shape factor distributions in CFD are predicted with the best overall accuracy for the highest inlet velocity, $18 \mathrm{~m} / \mathrm{s}$. At two cases of lower inlet velocity, the transition occurs earlier in CFD. For the case of $9 \mathrm{~m} / \mathrm{s}$ inlet velocity, 


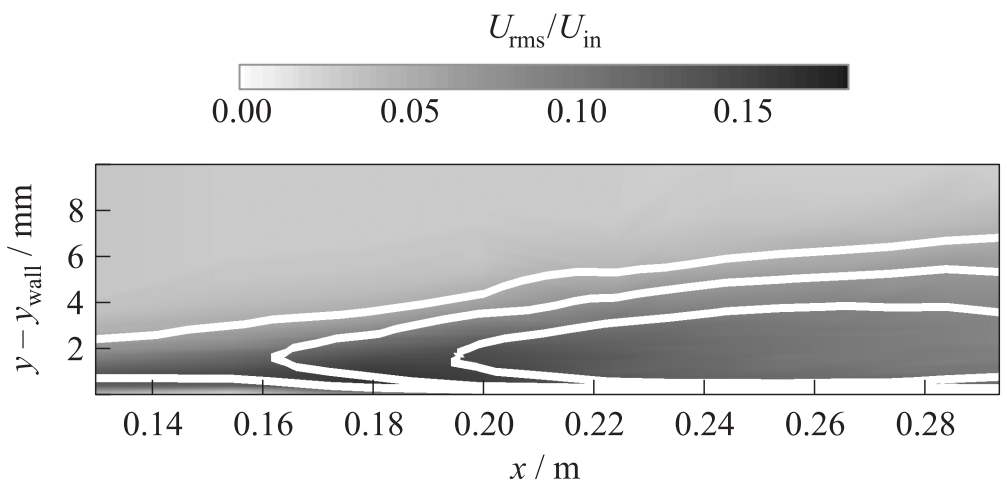

(a)

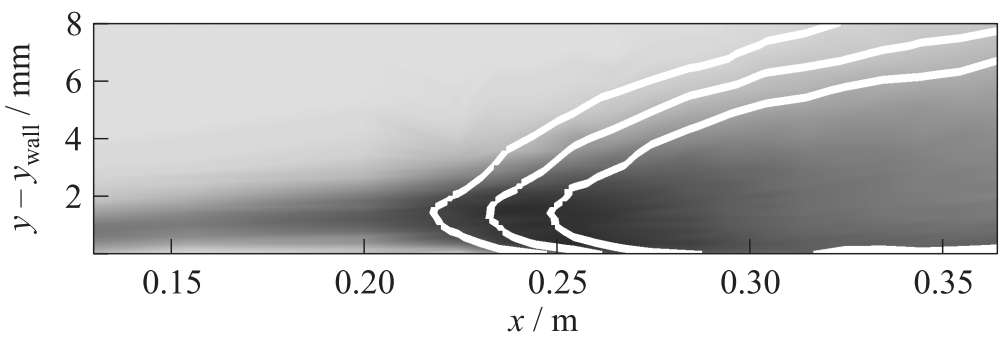

(b)

Figure 8 Effect of the inlet turbulence intensity for Case 4 at $U_{\text {in }}=9 \mathrm{~m} / \mathrm{s}:(a)$ the case of $\mathrm{Tu}_{\mathrm{in}}=4 \%$ and $(b) \mathrm{Tu}_{\mathrm{in}}=2 \%$. Pseudocolors depict local turbulence intensity $U_{\text {rms }} / U_{\text {in }}$ and white contour lines depict intermittency levels of $0.25,0.5$, and 0.75 . Note different streamwise axes

the transition in CFD starts already at the beginning of the shown interval, at $x=0.15 \mathrm{~m}$, while in the experiment, the transition begins at $x=0.2 \mathrm{~m}$. The transition location is overpredicted by $0.05 \mathrm{~m}$, which would correspond to $10 \%$ of the cord assuming the equivalent chord of $0.5 \mathrm{~m}$. In experiment, the transition occurs at the end of the zone with adverse pressure gradient and in CFD at the beginning of this zone, just downstream of the pressure minimum. For the case of $5 \mathrm{~m} / \mathrm{s}$, the transition in CFD starts at $x=0.2 \mathrm{~m}$, while in the experiment, the transition begins at $x=0.27 \mathrm{~m}$. The transition location overprediction is $0.07 \mathrm{~m}$. In both CFD and experiment, the transition occurs at the end of the zone of adverse pressure gradient. One can conclude that the transition model predicts the transition location at low Reynolds numbers and mild pressure gradients in a conservative fashion. To trigger the transition earlier is a conservative approach, since at mild pressure gradient, the possibility of the flow separation is excluded, so that the friction drag is slightly overpredicted rather than underpredicted. 

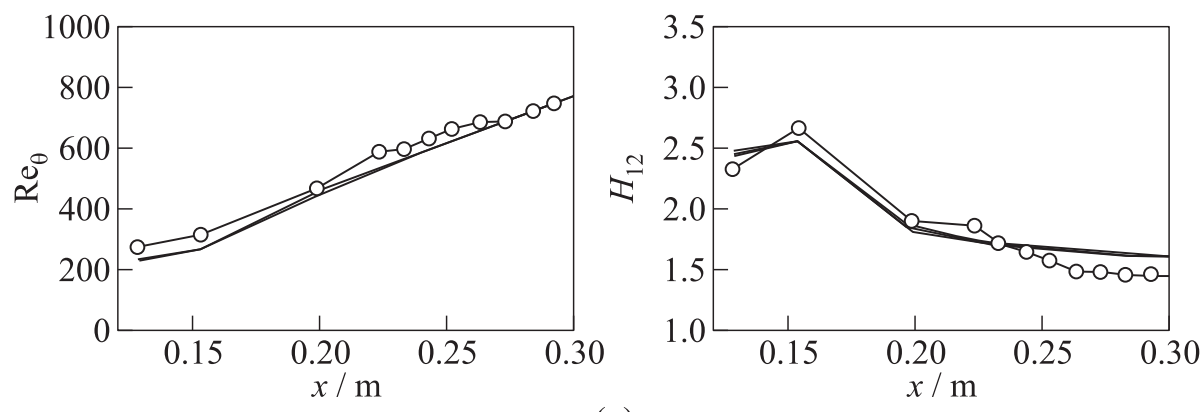

(a)
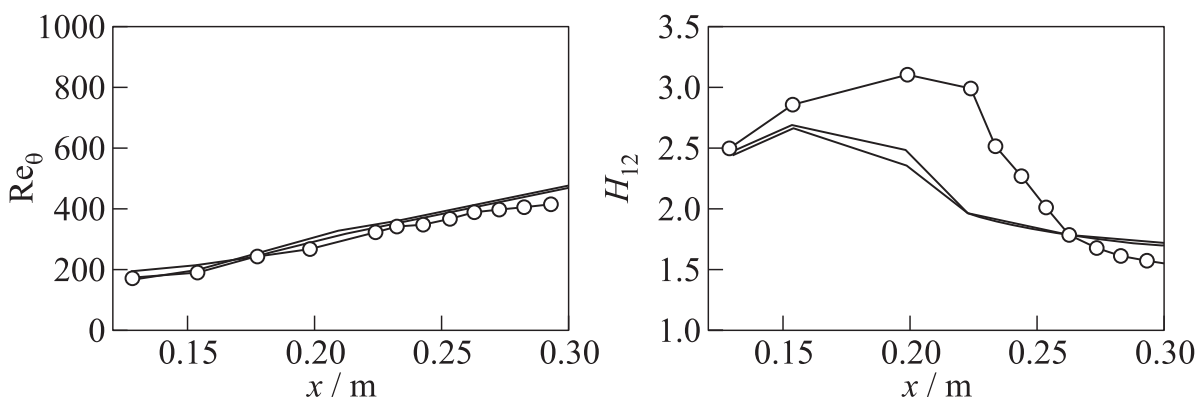

(b)
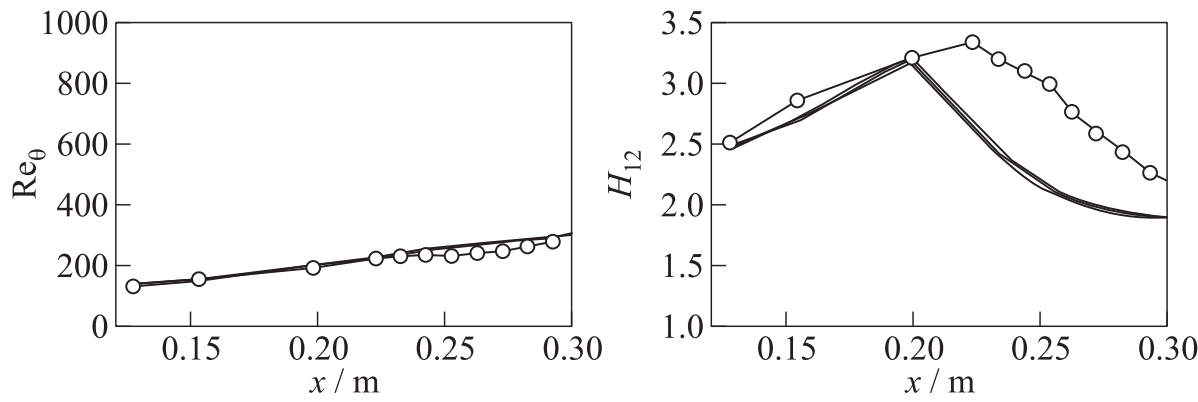

(c)

Figure 9 Comparison of experiment and $\mathrm{CFD}$ at $\mathrm{Tu}_{\text {in }}=2 \%$ for the case of mild pressure gradient (Case 4) and $U_{\text {in }}=18(a), 9(b)$, and $5 \mathrm{~m} / \mathrm{s}(c)$. Symbols show experimental data and lines are the CFD results on 3 different meshes

One can note that the experimental values of the shape factor agree with anticipated values expected from the pressure distributions and demonstrate reasonable behavior. Particularly, at the pressure minimum, the shape factor is close to the shape factor for the Blasius boundary layer which is 2.59. Through the measurement region, the shape factor is below the value for the laminar 
separation which is 3.85 and at the end of the measured region for the highest Reynolds-number case, the shape factor is approaching the value for the developed turbulent boundary layer on a flat plate, which is 1.3 .

Experimental transition locations in terms of $\operatorname{Re}_{\theta}$ are, respectively, 200, 250, and 300 for the velocities 5,9 , and $18 \mathrm{~m} / \mathrm{s}$. The local acceleration parameter, $K=\left(\nu / U^{2}\right)(d U / d x) \cdot 10^{6}$ is $-2,-1.2$, and -1.0 , respectively. In CFD, the
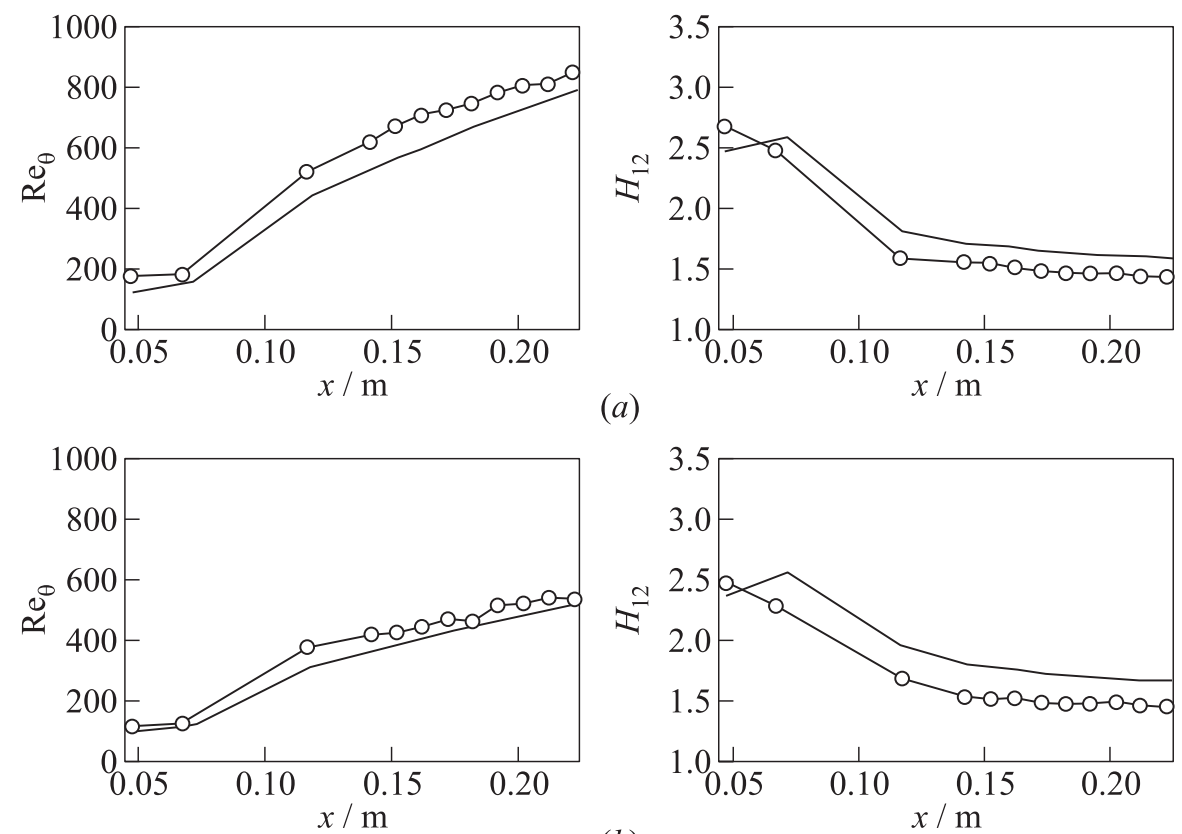

(b)
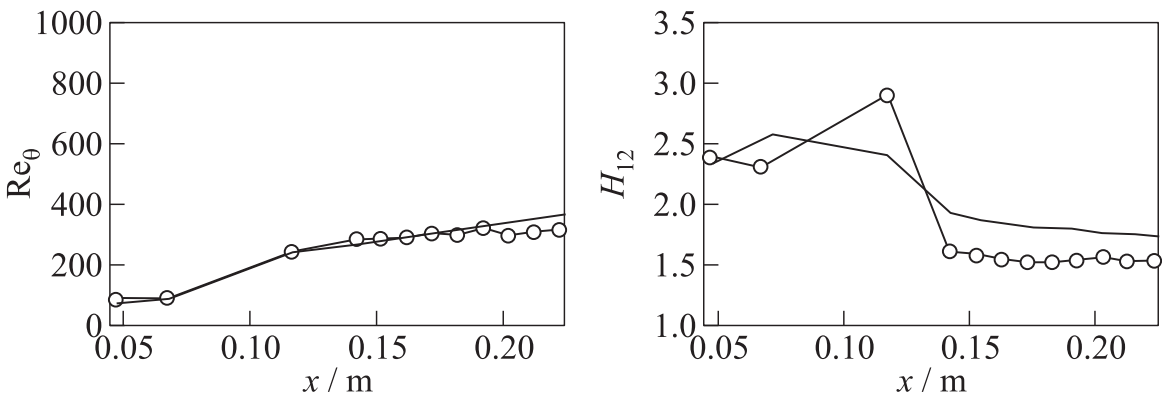

(c)

Figure 10 Comparison of experiment (symbols) and CFD (curves, results from a medium-size grid calculations) at $\mathrm{Tu}_{\mathrm{in}}=2 \%$ for the case of strong pressure gradient (Case 2) and $U_{\text {in }}=18(a), 9(b)$, and $5 \mathrm{~m} / \mathrm{s}(c)$ 
transition occurs for $\operatorname{Re}_{\theta}=200$ in all cases. The results of current experiments agree well with earlier reported values of transition Reynolds number 250-300 [1, $3]$ for 2 percent turbulence intensity.

In the case of stronger pressure gradient, the results are shown in Fig. 10. The agreement of CFD and experiment is very good for $\operatorname{Re}_{\theta}$ and $H_{12}$ distributions. The transition occurs at $x=0.07 \mathrm{~m}$ both in CFD and in experiment.
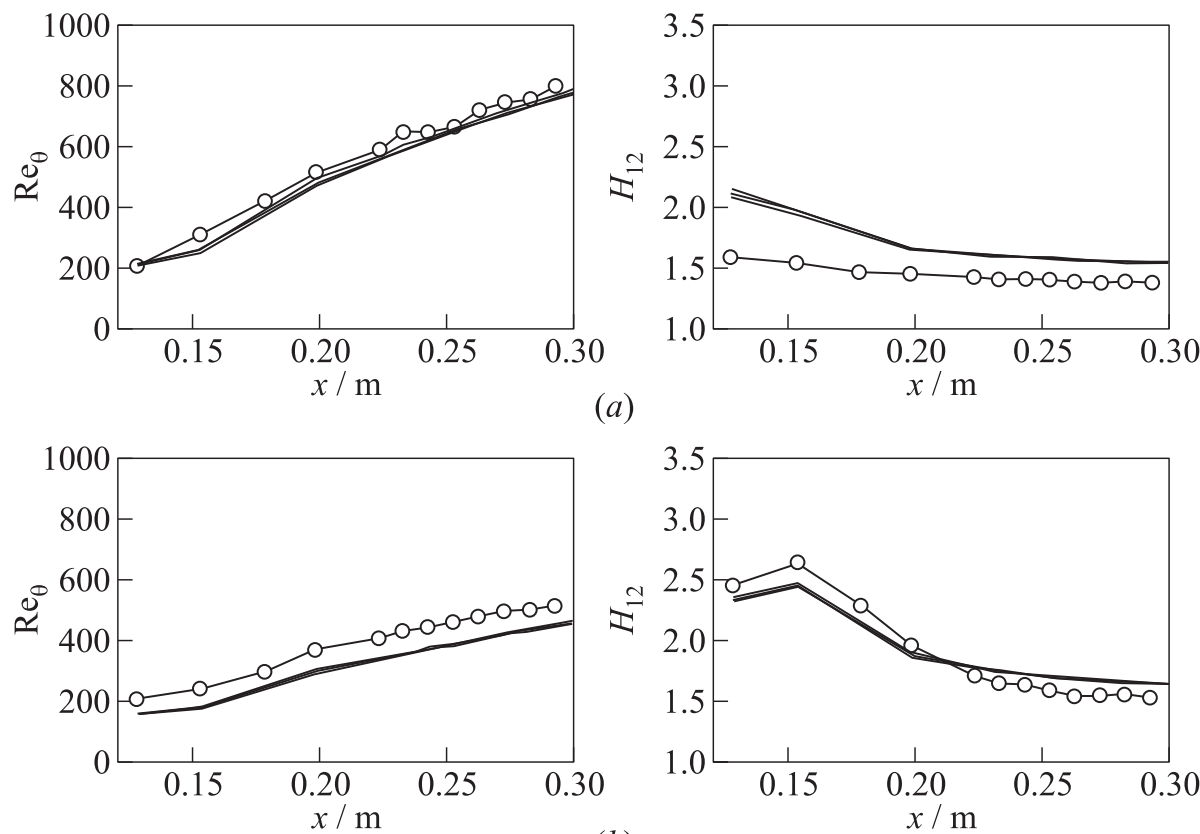

(b)
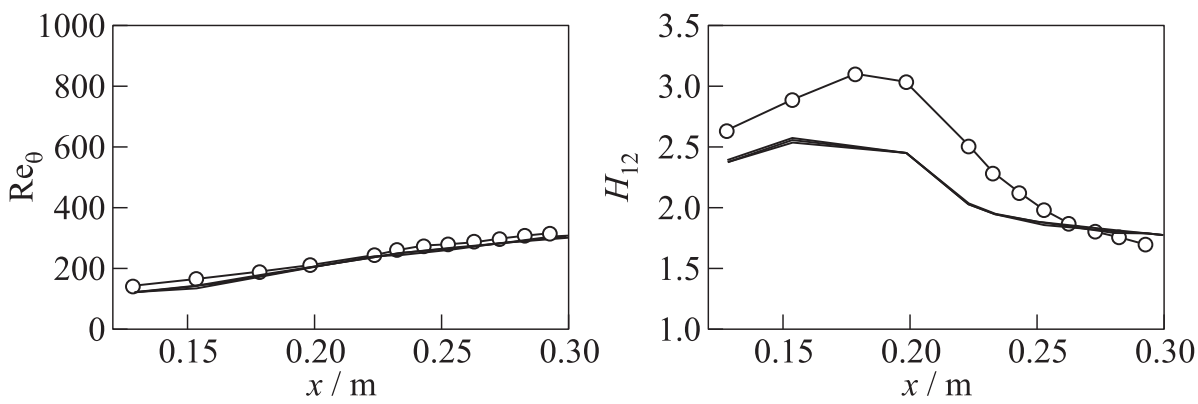

(c)

Figure 11 Comparison of experiment (symbols) and CFD (curves, results on 3 different meshes) at $\mathrm{Tu}_{\mathrm{in}}=4 \%$ for the case of mild pressure gradient (Case 4) and $U_{\text {in }}=18(a), 9(b)$, and $5 \mathrm{~m} / \mathrm{s}(c)$ 
This is the location just behind the pressure minimum where the flow separates. The transition Reynolds number from experiments is 100, 150, and 200 for the velocities 5,9 , and $18 \mathrm{~m} / \mathrm{s}$. In this case, the separation-induced transition takes place and the acceleration parameter, $K$, is $-9,-5$, and -3 .

For the cases with the turbulence intensity elevated to $4 \%$, the results are presented in Fig. 11. In this case, the CFD results are shown from 3 different numerical grids to demonstrate the grid independency. The development of the momentum thickness Reynolds number is captured very well by CFD in all cases. Again, some observed discrepancies are caused, mainly, by differences in the values of the momentum thickness in experiment and CFD. The experimental values of the momentum thickness are about $50 \mu \mathrm{m}$ larger, which again is explained by the accuracy of the hot-wire positioning with respect to the wall. The shape factor distributions are predicted the best for the medium inlet velocity, $9 \mathrm{~m} / \mathrm{s}$.

For the case of the lowest inlet velocity, $5 \mathrm{~m} / \mathrm{s}$, the transition occurs earlier in CFD by about $0.03 \mathrm{~m}$, which would correspond to $6 \%$ of chord at equivalent chord of $0.5 \mathrm{~m}$. Again, the turbulence model demonstrates a conservative behavior similarly to Case 4 discussed above.

For the case of $18 \mathrm{~m} / \mathrm{s}$, the transition location is underpredicted by about $0.05 \mathrm{~m}$. The experimental data do not extend far enough upstream to make a more precise conclusion. At $18 \mathrm{~m} / \mathrm{s}$, both in CFD and in experiment, the transition starts in the zone of the favourable pressure gradient. However, in this case, the turbulence model demonstrates an optimistic behavior, which is undesirable, since it can lead to unsafe and misleading underprediction of the friction drag. The transition Reynolds number in experiment is around 200 for the two low-velocity cases (and presumably around 200 at $18 \mathrm{~m} / \mathrm{s}$ ) which agrees well with the reported in $[1,3]$ interval 150-200. The acceleration parameter, $K$, is below -2.5 at $5 \mathrm{~m} / \mathrm{s}$ (separation-induced transition), equal to -2 at $9 \mathrm{~m} / \mathrm{s}$, and presumably equal to 0.5 at $18 \mathrm{~m} / \mathrm{s}$. Since measurement data are missing for the upstream locations at $18 \mathrm{~m} / \mathrm{s}$, to perform additional measurements at high Reynolds number and high turbulence intensity is of great interest for the future work.

Nevertheless, the conclusions of the current paper are not affected anyhow and fully supported by the present data set.

\section{CONCLUDING REMARKS}

Laminar-turbulent transition at high free-stream turbulence in boundary layers of the airfoil-like geometries with the presence of the external pressure gradient changeover has been studied numerically and validated by experimental data. The comparison is performed for a number of flow cases with different flow Reynolds number, turbulence intensity, and pressure distributions. The flow parameters selected are typical for turbomachinery applications. 
The numerical calculations by using SST model with transition by Langtry and Menter show encouraging results:

- for the cases with strong pressure gradient, CFD demonstrates very good prediction of transition location for turbulence levels $2 \%$ and $4 \%$;

- in the case of mild pressure gradient, CFD computations demonstrate reasonably good predictions with some under- or overprediction of the transition onset;

- at 2 percent turbulence intensity and mild pressure gradient, the transition model shows conservative results at the middle and high flow Reynoldsnumber range. The transition is predicted earlier by $10 \%-15 \%$ of equivalent chord length;

- at high Reynolds number, the transition is predicted well;

- at 4 percent turbulence intensity, mild pressure gradient, and low Reynoldsnumber range, the transition model also demonstrates conservative results. The transition is predicted earlier by $6 \%$ of equivalent chord length;

- at the same other conditions and medium Reynolds number, the transition location is predicted very well; and

- for high Reynolds number, 4 percent turbulence intensity, and mild pressure gradient, the turbulence model demonstrates an optimistic behavior with transition predicted about $10 \%$ later than in experiments.

\section{REFERENCES}

1. Mayle, R.E. 1991. The 1991 IGTI Scholar Lecture: The role of laminar-turbulent transition in gas turbine engines. ASME J. Turbomach. 113:509-537.

2. Abu-Ghannam, B. J., and R. Shaw. 1980. Natural transition of boundary layers the effects of turbulence, pressure gradient, and flow history. IMechE J. Mech. Eng. Sci. 22:213-228.

3. Blair, M. F. 1982. Influence of free-stream turbulence on boundary layer transition in favourable pressure gradients. J. Eng. Power 104:743-750.

4. Walker, G. J., and J. P. Gostelow. 1990. Effects of adverse pressure gradients on the nature and length of boundary-layer transition. ASME J. Turbomach. 112:196-205.

5. Gostelow, J. P., A. R. Blunden, and G. J. Walker. 1994. Effects of freestream turbulence and adverse pressure gradients on boundary-layer transition. ASME J. Turbomach. 116:392-404.

6. Solomon, W. J., G. J. Walker, and J. P. Gostelow. 1996. Transition length prediction for flows with rapidly changing pressure gradients. ASME J. Turbomach. 118:744751. 
7. Boiko, A. V., A. V. Dovgal, G. R. Grek, and V. V. Kozlov. 2012. Physics of transitional shear flows. Dordrecht-Heidelberg-London-New York: Springer. 271 p.

8. Menter, F. R., R. B. Langtry, S. R. Likki, Y. B. Suzen, P. G. Huang, and S. Völker. 2004. A correlation based transition model using local variables. Part 1. Model formulation. ASME Paper No. GT2004-53452.

9. Langtry, R. B., F. R. Menter, S. R. Likki, Y. B. Suzen, P. G. Huang, and S. Völker. 2004. A correlation based transition model using local variables. Part 2. Test cases and industrial applications. ASME Paper No. GT2004-53454.

10. Chernoray, V., S. Ore, and J. Larsson. 2010. Effect of geometry deviations on the aerodynamic performance of an outlet guide vane cascade. ASME Paper No. GT2010-22923.

11. Johansson, M., V. Chernoray, L. Ström, J. Larsson, and H. Abrahamsson. 2011. Experimental and numerical investigation of an aerodynamically loaded guide vane in a turbine duct. ASME Paper No. GT2011-46221.

12. Niebles Atencio B. M. 2011. Measurements and prediction of laminar-turbulent transition at high free-stream turbulence in boundary layers with pressure gradients. Gothenburg: Chalmers University of Technology. M.Sc. Thesis. 41 p. 